\title{
Iridescent colouration of male Anna's hummingbird (Calypte anna) caused by multilayered barbules
}

\author{
Marco A. Giraldo ${ }^{1} \cdot$ Juan L. Parra ${ }^{2} \cdot$ Doekele G. Stavenga $^{3}$ D
}

Received: 3 March 2018 / Revised: 16 September 2018 / Accepted: 26 September 2018 / Published online: 8 October 2018

(c) The Author(s) 2018

\begin{abstract}
The male Anna's hummingbird features a brightly reddish-pink reflecting gorget, due to large stacks of melanosomes in the feather barbules, arranged in layers separated by keratin. Direct observations together with detailed scatterometry demonstrated that the barbules reflect incident light in an approximately specular manner. The structural colouration is iridescent, i.e. varies with a changing angle of light incidence. Spectrophotometrical measurements of the barbule reflectance and absorbance can be well interpreted with calculated spectra obtained with a transfer matrix method for optical multilayers, using anatomical data and measured refractive index spectra. The organization of the reflectors as a Venetian blind presumably functions to create a high spectral contrast of the male's plumage during courtship.
\end{abstract}

Keywords Feather iridescence $\cdot$ Courtship $\cdot$ Spectrophotometry $\cdot$ Scatterometry $\cdot$ Optical modelling

\section{Introduction}

Among the many colourful birds, hummingbirds stand out because of their extremely shiny feathers (Greenewalt 1960; Stoddard and Prum 2011; Cuthill et al. 2017). Especially male hummingbirds feature brilliant plumages, which they display during courtship toward females. The male's colourful plumage, as well as his courtship behaviour, is presumably a product of aesthetic evolution by female choice (Darwin 1871; Prum 2012). The iridescent ornamental feathers are sensitive to diet quality and may serve as honest signals of nutrition to mates or rivals (Doucet and Meadows 2009; Meadows et al. 2012).

The barbules of the brightly reflecting feathers of hummingbirds contain stacks of melanosomes,

Doekele G. Stavenga

D.G.Stavenga@rug.nl

1 Biophysics Group, Institute of Physics, University of Antioquia, Calle 70 \#52-21, AA 1226, Medellín, Colombia

2 Group of Ecology and Evolution of Vertebrates, Institute of Biology, University of Antioquia, Calle 70 \#52-21, AA 1226, Medellín, Colombia

3 Computational Physics, Zernike Institute for Advanced Materials, University of Groningen, Nijenborgh 4, 9747 AG Groningen, The Netherlands melanin-containing organelles with large air spaces (Greenewalt et al. 1960a). The barbule material thus has a strongly varying refractive index, resembling that of a multilayered interference reflector (Durrer 1977). Optical modelling on the Fiery topaz (Topaza pyra) and Anna's hummingbird (Calypte anna) showed that measured reflectance spectra could be well interpreted as to be created by an optical, dielectric multilayer (Greenewalt et al. 1960a, b). At that time, however, the refractive indices of the main material components of the barbules, keratin and melanin, were ill-known. The refractive index values were assumed to be real and wavelength-independent, not in accordance with the complex refractive index of the strongly absorbing melanin.

Recent measurements on various bird feathers have yielded the data necessary for a detailed analysis of bird structural colouration (Leertouwer et al. 2011; Stavenga et al. 2015). Here we focus on the male Anna's hummingbird (C. anna), which has a strikingly coloured, reddish-pink crown and gorget. The colouration is strongly iridescent, because it is very dependent on the angle of illumination and observation (Doucet and Meadows 2009; Meadows et al. 2011). We performed spectrophotometry, scatterometry, anatomy, and optical modelling to investigate the colouration of the gorget feathers. Our study extends previous treatises, confirming that the melanosome stacks essentially behave as an optical multilayer. 


\section{Materials and methods}

\section{Animals and microphotography}

Feathers of the gorget of Anna's hummingbird (C. anna) were obtained from the Museum of Vertebrate Zoology (UC Berkeley; sample size 7 feathers). Intact feathers were photographed with an Olympus stereoscope (SZX16 Stereo Zoom Microscope) and an Olympus SC-30 digital camera. Single barbs, mounted on a rotatable stage, were photographed with a Zeiss Universal Microscope (Zeiss, Oberkochen, Germany) using Zeiss Epiplan objectives $(16 \times / 0.35$ or $40 \times / 0.85)$ and a Kappa DX-40 (Kappa Optronics, Gleichen, Germany) digital camera. Images of barbs with barbules in oil immersion were taken using a Zeiss $100 \times$ oil (NA 0.9) objective.

\section{Spectrophotometry}

Reflectance spectra of the distal, iridescent part of single feathers were recorded with a bifurcated reflection probe (Avantes, Apeldoorn, the Netherlands) using a deuterium-halogen lamp (Avantes AvaLight-D(H)-S) and an AvaSpec-2048 spectrometer (Avantes). A white reflectance standard (WS-2, Avantes) served as a reference. Reflectance spectra were also measured from single barbules with a home-built microspectrophotometer (MSP), which consists of a Leitz Ortholux epi-illumination microscope connected with a fiber optic to the AvaSpec-2048 spectrometer. The light source was a xenon arc and the microscope objective was an Olympus LUCPlanFL N 20×/0.45. Due to the glass UV-absorption, the MSP spectra were limited to wavelengths $>350 \mathrm{~nm}$. Transmittance spectra were measured with the MSP from barbules immersed in oil to reduce light scattering, and were subsequently converted into absorbance spectra.

\section{Imaging scatterometry}

To investigate the spatial far-field reflection properties, we performed imaging scatterometry on small pieces of barbs with about ten barbules on each side (Stavenga et al. 2009). The pieces, attached to the tip of a glass micropipette, were positioned at the first focal point of the ellipsoidal mirror of the imaging scatterometer. Scatterograms were obtained by focusing a white light beam with a narrow aperture $\left(<5^{\circ}\right)$ onto a circular spot with a diameter of $\sim 30 \mu \mathrm{m}$ to illuminate isolated barbules. The spatial distribution of the far-field scattered light was recorded with an Olympus DP70 digital camera (Olympus, Tokyo, Japan).

\section{Electron microscopy}

A Philips XL-30 scanning electron microscope (SEM) was used to investigate the structure of the barbules and their arrangement on the barbs. To reveal the morphology of the barbules, they were transversally cut with a razor blade, placed on a carbon stub holder, and then sputtered with gold (5-10 nm thickness). For transmission electron microscopy we cut feather barbs from the distal portion of a gorget feather and incubated them in $0.25 \mathrm{M}$ sodium hydroxide and $0.1 \%$ Tween 20 for $30 \mathrm{~min}$ on a bench-top shaker. These barbs were then transferred to a $2: 3(\mathrm{v} / \mathrm{v})$ solution of formic acid and ethanol for $2.5 \mathrm{~h}$. Feather barbs were dehydrated by incubating in $100 \%$ ethanol twice $(20 \mathrm{~min})$ and $100 \%$ propylene oxide once $(20 \mathrm{~min})$ and infiltrated with Epon 812 in successive concentrations of 15, 50, 70 (48 h each) and $100 \%$ (24 h). We placed the barb sections into molds with the most distal tip of the barb at the top of the mold, and then the blocks were cured in an oven at $20^{\circ} \mathrm{C}$ for $24 \mathrm{~h}$. Finally, we sliced the molds using a diamond knife on an RMC MT-X ultramicrotome (Boeckeler Instruments, Tucson, AZ) to obtain transversal sections relative to the barb. Sections were placed on 200 mesh copper grids (EMS, Fort Washington, PA) with formvar support, post-stained in osmium and lead citrate, and viewed on a Philips EM301 (Veeco FEI Inc, Hillsboro, OR) between 6000 and 60.000× magnification.

\section{Optical modelling}

We calculated the reflectance and absorbance spectra of model barbules with a transfer matrix program based on classical optical multilayer theory, written in Matlab (Stavenga 2014). The model barbules were stacks of melanosomes, embedded in a keratin matrix (Fig. 6c). Based on the anatomy, we used the following parameter values: thickness of the melanosome membrane $a=30 \mathrm{~nm}$; thickness internal air layer of top melanosomes $b_{1}=20-50 \mathrm{~nm}$; thickness internal air layer of other melanosomes $b_{2}=100-125 \mathrm{~nm}$, thickness keratin cortex $c=5 \mathrm{~nm}$; thickness keratin layer in between melanosomes $d=50 \mathrm{~nm}$; number of melanosome layers was generally taken to be 12 , except for Fig. 8c where the number was varied. In the melanosome layers, each melanosome plus surrounding keratin was assumed to occupy a square with area $(1000 \mathrm{~nm})^{2}$, with an outside keratin fraction $f_{\mathrm{k}}=0.05$, or, the melanosome area was a square with side $w=975 \mathrm{~nm}$. Thin walls inside the melanosomes, perpendicular to the membrane surface, with thickness $e=10 \mathrm{~nm}$, divided the internal space into air chambers. Taking $N=10$ internal walls in a cross-section, or a mesh of $(N+1)^{2}=121$ square air holes, the resulting fractions of melanin and air in the internal layer were $f_{\mathrm{m}}=0.40$ and $f_{\mathrm{a}}=0.55$. The real parts 
of the wavelength-dependent refractive indices of the two components, $n_{\mathrm{k}}$ and $n_{\mathrm{m}}$, were calculated with the Cauchy formula $n=A+B \lambda^{-2}$ ( $\lambda$ is the light wavelength), using for keratin $A_{\mathrm{k}}=1.532$ and $B_{\mathrm{k}}=5890 \mathrm{~nm}^{-2}$ and for melanin $A_{\mathrm{m}}=1.648$ and $B_{\mathrm{m}}=23,700 \mathrm{~nm}^{-2}$; the imaginary component of the refractive index of keratin was assumed to be negligible in the wavelength of interest, but that of melanin was taken to be $k_{\mathrm{m}}=a_{\mathrm{m}} \exp \left(-\lambda / b_{\mathrm{m}}\right)$, with $a_{\mathrm{m}}=0.56$ and $b_{\mathrm{m}}=270 \mathrm{~nm}$ (Leertouwer et al. 2011; Stavenga et al. 2015). The barbule was sliced into $10 \mathrm{~nm}$ thick layers and together with the volume fractions of keratin $\left(f_{\mathrm{k}}\right)$, melanin $\left(f_{\mathrm{m}}\right)$ and air $\left(f_{\mathrm{a}}\right)$, with $f_{\mathrm{k}}+f_{\mathrm{m}}+f_{\mathrm{a}}=1$, the effective refractive index of each slice, and thus the barbule's refractive index profile, followed from

$n_{\text {eff }}=f_{\mathrm{k}} n_{\mathrm{k}}+f_{\mathrm{m}}\left(n_{\mathrm{m}}-i k_{\mathrm{m}}\right)+f_{\mathrm{a}} n_{\mathrm{a}}$.

\section{Results}

\section{Spectral and spatial characteristics}

The gorget and crown of $C$. anna are covered by bright, reddish-pink feathers (Fig. 1a). The colour is restricted to the distal feather part (Fig. 1b). An isolated gorget feather, positioned with the feather plane about perpendicular to the axis of the observation microscope, showed a maximal reflection when illuminated by a fiber light source from an angle of $40^{\circ}$, meaning that the reflecting elements are rotated by an angle of about $20^{\circ}$ with respect to the feather plane (Fig. 1b). The bright reflection almost fully vanished upon rotation of the feather around an axis perpendicular to the rachis over $-20^{\circ}$ (Fig. 1c) or $+20^{\circ}$ (Fig. 1d), thus demonstrating that the feather reflection is about specular, in full agreement with angle-dependent reflectance spectra measured from iridescent feathers of Anna's hummingbird by Meadows et al. (2011).

The reddish-pink colour indicates that the feather reflects red light as well as some blue. Measurement of the reflectance spectrum with a bifurcated probe indeed showed a main reflectance band in the red wavelength range, peaking at $\sim 670 \mathrm{~nm}$ (halfwidth $\sim 100 \mathrm{~nm}$ ), together with a minor band in the blue, peaking at $\sim 480 \mathrm{~nm}$ (Fig. 1e). Measurements with a microspectrophotometer from small $(10 \mu \mathrm{m})^{2}$ areas yielded very similar spectra, although the bandwidth was slightly narrower (Fig. 1e), demonstrating that the probe spectra are averages of somewhat variable local spectra.

Scatterometry of a small distal piece of the feather confirms the directionality of the reflection. A narrow-aperture, white light beam illuminating the feather piece about normally caused a spatially restricted, reddish-pink scatterogram (Fig. 2a). When the illumination's angle of incidence was increased, the specularity of the feather was maintained, but the colour changed to shorter wavelengths (Fig. 2b-k).
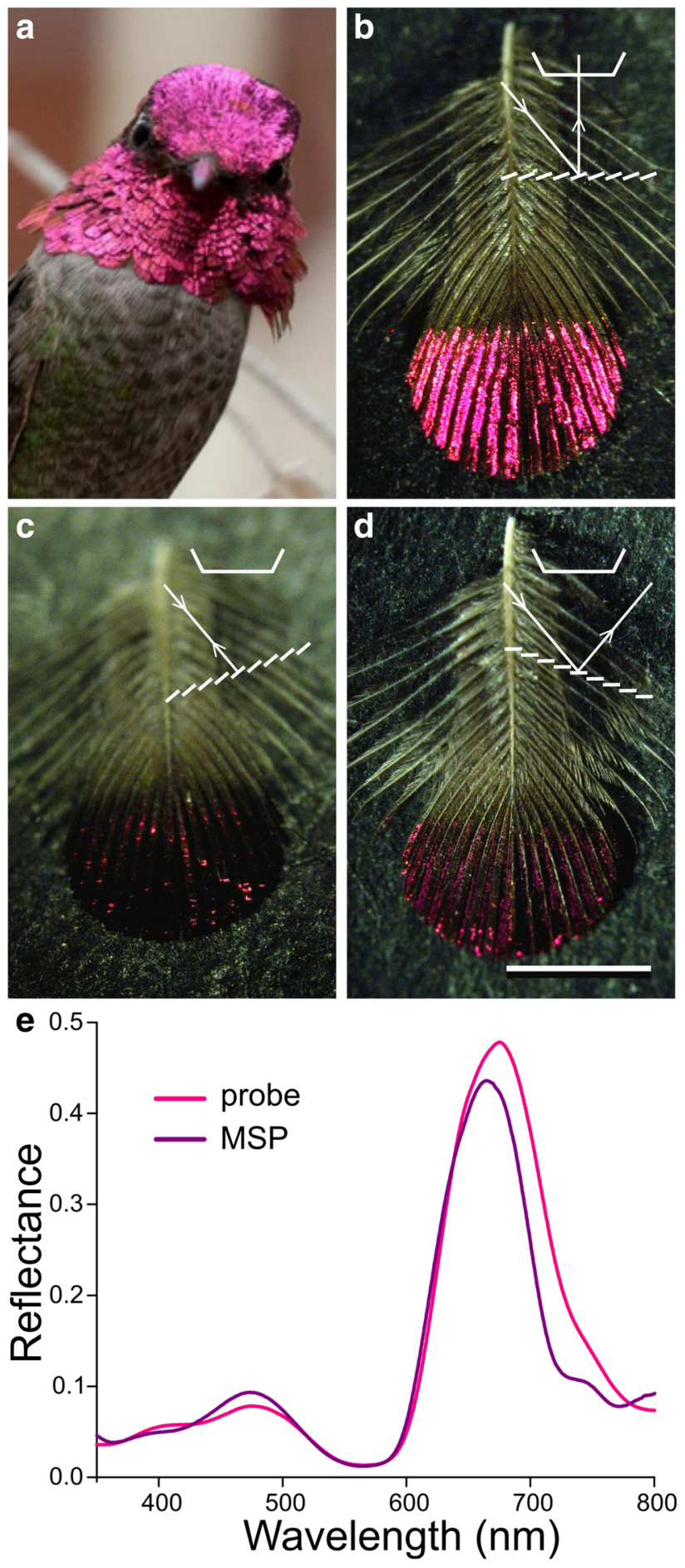

Fig. 1 The bright reddish-pink colouration of Anna's hummingbird. a The gorget and crown feathers feature the same colour. b An isolated gorget feather showing that only the distal part is coloured. c The same feather rotated over $-20^{\circ}$ around an axis perpendicular to the feather's rachis. $\mathbf{d}$ The feather rotated over $+20^{\circ}$; scale bar $(\mathbf{b}-\mathbf{d})$ : $2 \mathrm{~mm}$. Insets: diagrams of obliquely incident light reflected by the feather barbules into the aperture of the microscope objective (b) and outside the aperture $(\mathbf{c}, \mathbf{d})$. e Reflectance spectra of the distal area of an isolated feather measured with a bifurcated probe and with a microspectrophotometer (MSP) 

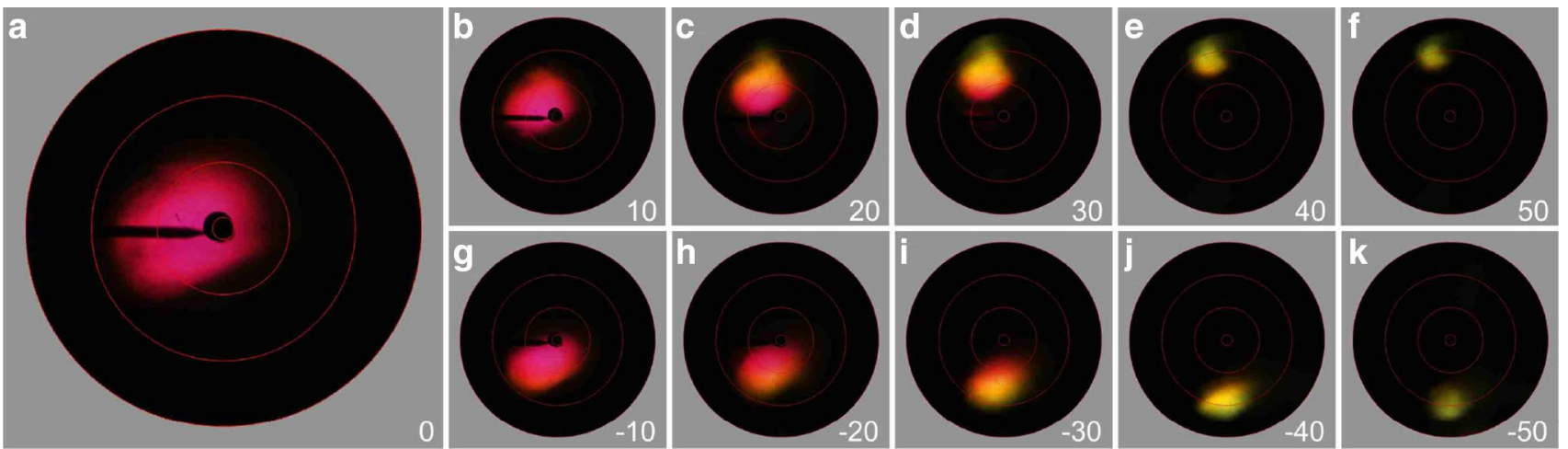

Fig. 2 Scatterometry of a small area of the distal part of the feather of Fig. 1b-d. a Scatterogram obtained from a slightly tilted $\left(\sim 20^{\circ}\right)$ feather applying a narrow-aperture, white-light beam causing reflection into about the axial direction $\left(\sim 0^{\circ}\right)$ of the scatterometer. b-f Scatterograms resulting from illuminations when the angle of inci-

Such a short-wavelength shift is characteristic for an optical multilayer, suggesting the presence of a multilayer-like structure in the hummingbird feathers.

\section{Structural colouration and pigmentation}

Inspection of the feathers with an epi-illumination microscope demonstrated that the coloured reflections emerge from the barbules (Fig. 3a). Observation with transmitted light yielded a very different picture, indicating a highly absorbing material in the barbules (Fig. 3b). To show that dence was changed in steps of $\sim 10^{\circ}$ upwards $\left(0^{\circ}-50^{\circ}\right)$. $\mathbf{g}-\mathbf{k}$ Scatterograms resulting from illuminations when the angle of incidence was changed in steps of $\sim 10^{\circ}$ downwards $\left(-10^{\circ}\right.$ to $\left.-50^{\circ}\right)$. The red circles indicate scattering angles of $5^{\circ}, 30^{\circ}, 60^{\circ}$, and $90^{\circ}$; the black bar at 9 o'clock is the shadow of the glass pipette holding the feather piece
Fig. 3 Micrographs of a feather piece in reflected and in transmitted light, and barbule absorbance. a Epi-illumination micrograph of a few barbs with barbules, showing highly red-pinkish reflecting barbules. b Transmitted-light micrograph showing very dark-brown barbules (in air). c Transmittedlight micrograph of barbules in oil immersion; scale bar $(\mathbf{a}-\mathbf{c})$ : $200 \mu \mathrm{m}$. d Absorbance spectra of two barbule locations and the absorbance spectrum of pure melanin
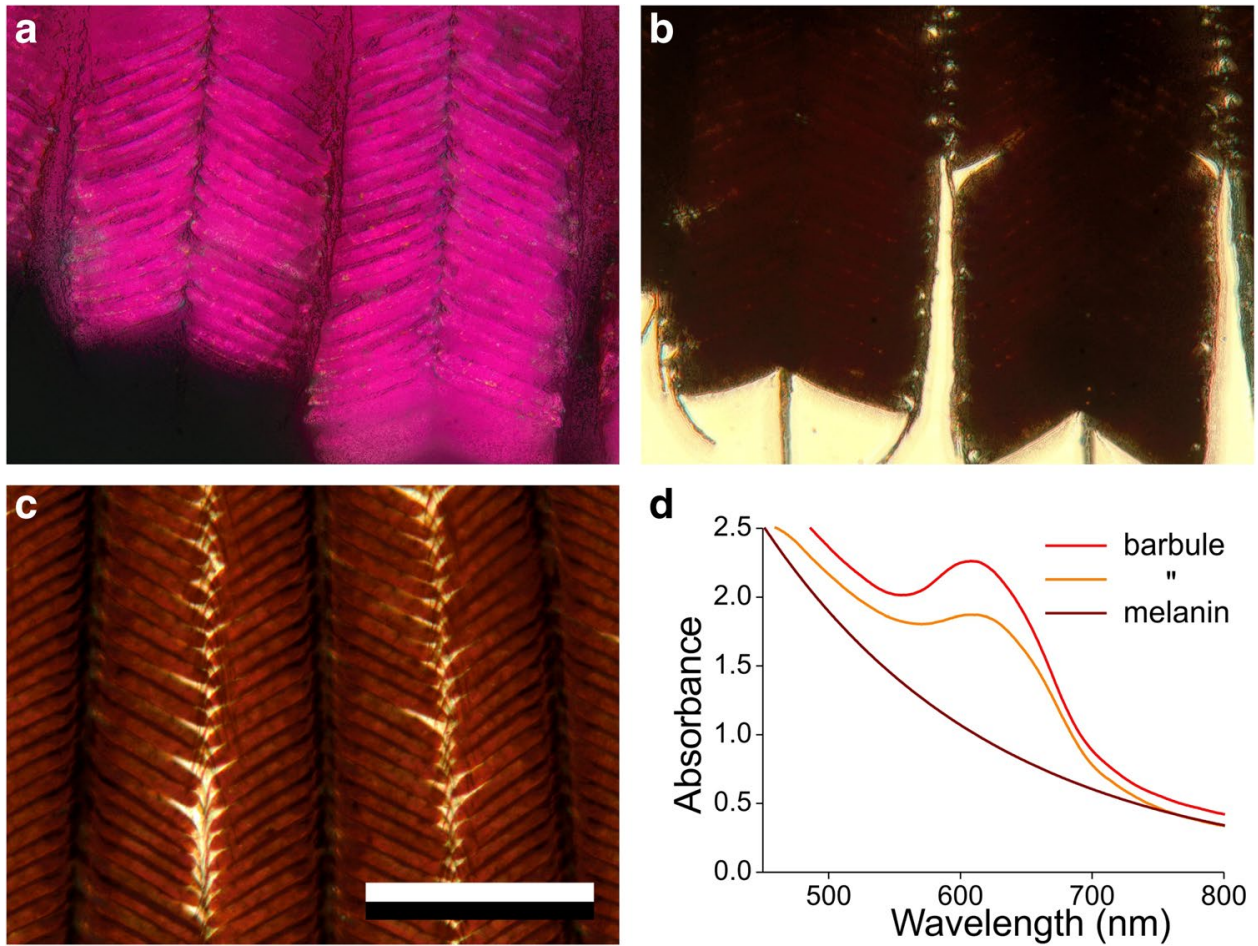
To ascertain that the steep increase in absorbance at the shorter wavelengths was indeed due to melanin, we considered that the absorbance of an object, thickness $d$, containing eumelanin with concentration $C$, equals $D(\lambda)=\varepsilon_{0} C d \exp \left(-\lambda / \lambda_{\mathrm{m}}\right)$, with $\varepsilon_{0}=2.45 \mu \mathrm{m}^{-1} \mathrm{M}^{-1}$, and $\lambda_{\mathrm{m}}=175 \mathrm{~nm}$ (Stavenga et al. 2012). We approximated the measured absorbance spectra of Fig. $3 d$ with the spectrum $D(\lambda)=33 \exp (-\lambda / 175)$. Assuming a concentration $C=7.9 \mathrm{M}$ of melanin, similar as previously derived for the densely pigmented wings of the male damselfly Calopteryx japonica (Stavenga et al. 2012), we obtain an effective thickness of $1.7 \mu \mathrm{m}$ for the pigment in the barbules of Anna's hummingbird gorget feathers. When the effective melanin thickness per melanosome is $97 \mathrm{~nm}$ (see "Materials and methods"), this would suggest that 17.5 layers of melanosomes exist. This value is in reasonable correspondence with the anatomy showing stacks of 12-15 orderly arranged melanosome layers together with a few additional, randomly organized melanosomes (see below).

We further investigated the structural properties of the barbules by isolating a single barbule and gluing it to the tip of a glass micropipette. The barbule appeared to be a long, longitudinally folded blade with a hook at the end. Only when properly oriented, epi-illumination of the barbule caused the bright reddish-pink colour (Fig. 4a, cf. Fig. 3a); in transmitted light the barbule was very darkbrown (Fig. 4b, cf. Fig. 3b, c). Upon rotation of the barbule over $80^{\circ}$, to expose the side lamina, epi-illumination created a broad-band whitish reflection (Fig. 4c), but in transmitted light the side lamina appeared to be rather transparent, with erratic interference colours, presumably due to thin film effects (Fig. 4d).

\section{Anatomy of the barbules of the male Anna's hummingbird}

The detailed shape and organization of the barbules became clear by performing electron microscopy. Scanning electron microscopy (SEM) demonstrated that the upper laminae of the barbules are angled with respect to the feather's plane, but parallel to each other as the lamellae in a Venetian blind (Fig. 5a). Both the exposed and side laminae have a length of $\sim 100 \mu \mathrm{m}$ and a width of $\sim 20 \mu \mathrm{m}$ (Fig. 5a,b, asterisks and arrowheads). The side sheet is prolongated into a more or less perpendicularly oriented hook with length $\sim 60 \mu \mathrm{m}$ (Fig. 5a,b, arrows). Close inspection showed that the upper lamina surface and its rim are marked by an irregular mosaic of spindle-shaped structures, length $1.1 \pm 0.2 \mu \mathrm{m}$ and width $0.24 \pm 0.04 \mu \mathrm{m}$ (Fig. $5 \mathrm{c}$ ). Cutting the barbules showed that the upper lamina consists of 12-15 layers, with periodicity $\sim 210 \mathrm{~nm}$, containing numerous holes (Fig. 5d).

The layers are especially clearly seen in transmission electron microscope (TEM) sections, revealing that the layers are
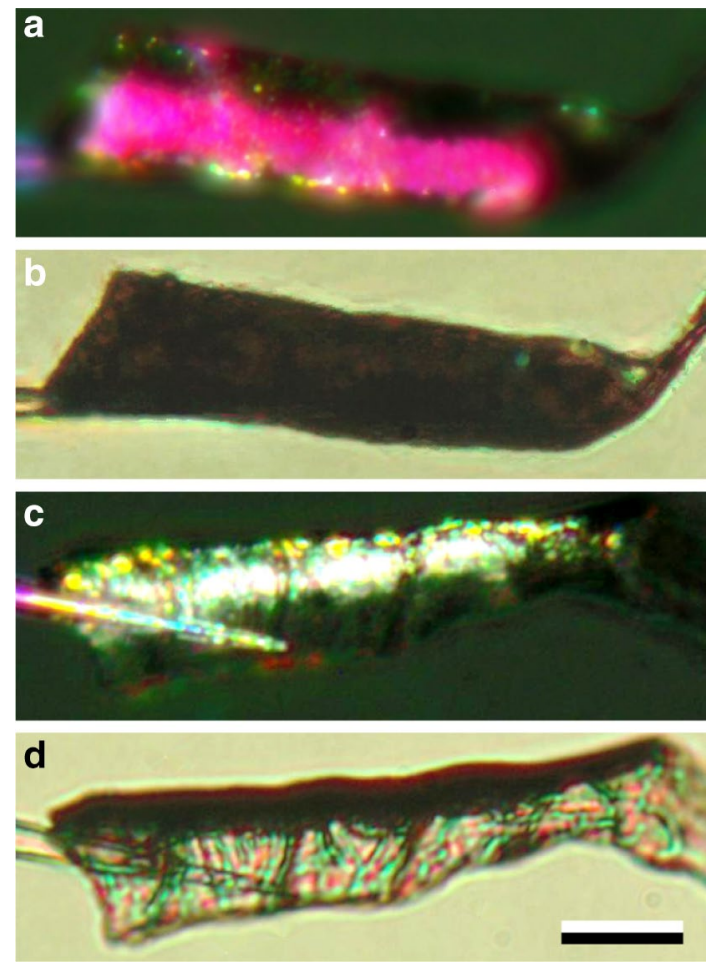

Fig. 4 An isolated barbule glued to a glass pipette. a Epi-illumination creating a bright reddish-pink reflection from the upper lamina. b The same situation but in transmitted light. $\mathbf{c}$ Epi-illumination of the barbule rotated by $80^{\circ}$. d The latter situation, but in transmitted light; scale bar (a-d): $20 \mu \mathrm{m}$

stacks of coplanar arranged melanosomes (Fig. 5e, f). The TEM micrographs show a very thin cortex. The upper layer of melanosomes is pressed against the cortex, explaining the corrugated barbule surface (Fig. 5c). Quite noticeably, the melanosomes in the upper layer are much thinner than the ones in the layers below (Fig. 5e, f), and furthermore SEM micrographs also indicated that their surface area is distinctly smaller than that of the lower ones (e.g. Fig. 5d). The period of the melanosome stack shown in the TEM images is $300-330 \mathrm{~nm}$ (Fig. 5e, f), much higher than the value $\sim 210 \mathrm{~nm}$ derived by SEM (Fig. 5d). Because optical modeling (see below) yielded period values of $210-230 \mathrm{~nm}$, in agreement with the SEM data, we conclude that the TEM sections were skew to the barbule surface.

To study the arrangement of the melanosomes more directly, we performed epi-illumination of small feather pieces immersed in oil (Fig. 6a). The reflection images showed a rather disordered pattern, similar as the SEM images (Fig. 5c). The bright areas evidently represented reflecting melanosomes, but in between numerous dark spots existed, indicating interstitial gaps. The pattern of the light micrograph is well compatible with Durrer's diagram (Fig. 6b) of the inner workings of hummingbird barbules (Durrer 1977). We have abstracted this spatial diagram into 
Fig. 5 Electron microscopy of hummingbird barbules. a Scanning electron micrograph of barbules attached to both sides of a barb. Each barbule is a folded plane with an exposed lamina (asterisk) and a pronounced (hidden) side wall (arrowheads), and at the end a long hook (arrow). b A single barbule showing the lamina (asterisk), side wall (arrowheads) and hook (arrow). c Both the top and lateral surfaces are marked by a mosaic consisting of spindle-shaped structures. d A cut barbule revealing layers with air holes in the main lamina. $\mathbf{e}$ Transmission electron micrograph of the transition edge area of the upper lamina and the side wall, showing numerous layers of melanosomes. $\mathbf{f}$ The upper layers of melanosomes, within the uppermost layer much smaller melanosomes than those in the other layers; scale bars: a $50 \mu \mathrm{m}, \mathbf{b} 20 \mu \mathrm{m}, \mathbf{c}-\mathbf{e} 2 \mu \mathrm{m}, \mathbf{f}$ $1 \mu \mathrm{m}$
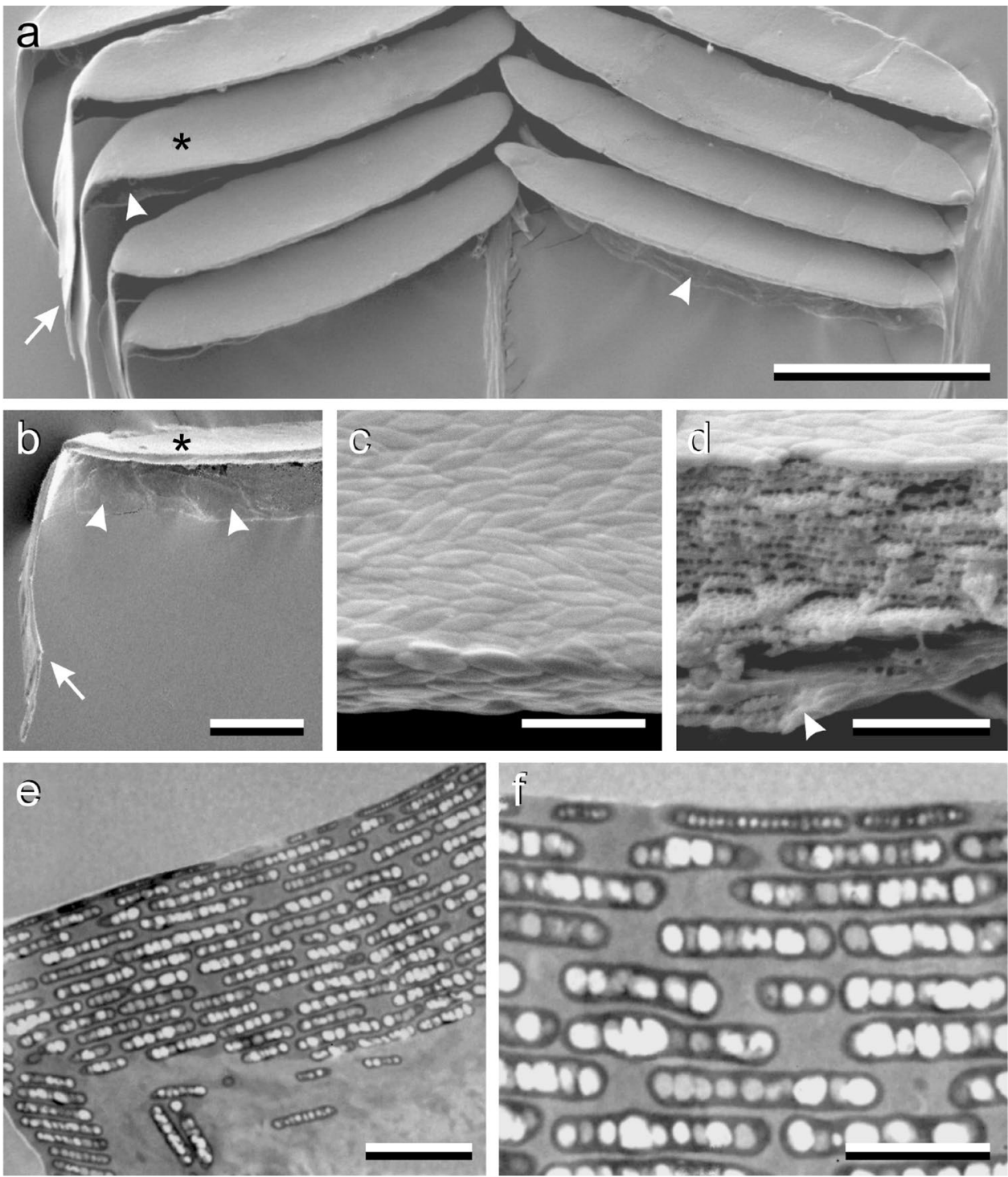

the model barbule of Fig. 6c, which we used in the computational modeling presented below.

\section{Modelling the barbule's structural colouration}

We calculated the reflectance and absorbance spectra for normally incident light, considering five special cases of barbules (Fig. 7). In case 1, we studied an idealized barbule with 12 layers of melanosomes. A thin $(5 \mathrm{~nm})$ cortex layer of pure keratin covered the layers containing melanosomes, with membrane thickness $30 \mathrm{~nm}$ and in between $100 \mathrm{~nm}$ thick air compartments. The melanosome layers were identical, except for the upper melanosome layer, where the melanosomes had $50 \mathrm{~nm}$ thick air compartments. A keratin layer of $50 \mathrm{~nm}$ separated the melanosome layers, so that the stack period was $210 \mathrm{~nm}$ (Fig. 6c). We calculated the effective refractive index along a coordinate normal to the barbule surface, using previously determined refractive index spectra, as described in "Materials and methods". The refractive index of the keratin cortex and the sheets in between the melanosome layers was real, but because the melanosome membranes as well as their internal walls contained melanin, the effective refractive index of the melanosome layer was complex (Fig. 7a, b). We implemented the above parameters in a transfer matrix program and calculated the reflectance spectrum as a function of wavelength, resulting in a spectrum with a distinct peak in the red and a broadband in the blue wavelength range (Fig. 7c). The simultaneously calculated absorbance spectrum also peaked in the red, although hypsochromic shifted (towards shorter wavelengths), and the absorbance sharply increased with decreasing wavelength, as expected (Fig. 7e, f, cf. Fig. 3d).

The anatomy of Fig. 5 clearly shows that neither the cortex surface nor the melanosome layers are perfectly flat, and 

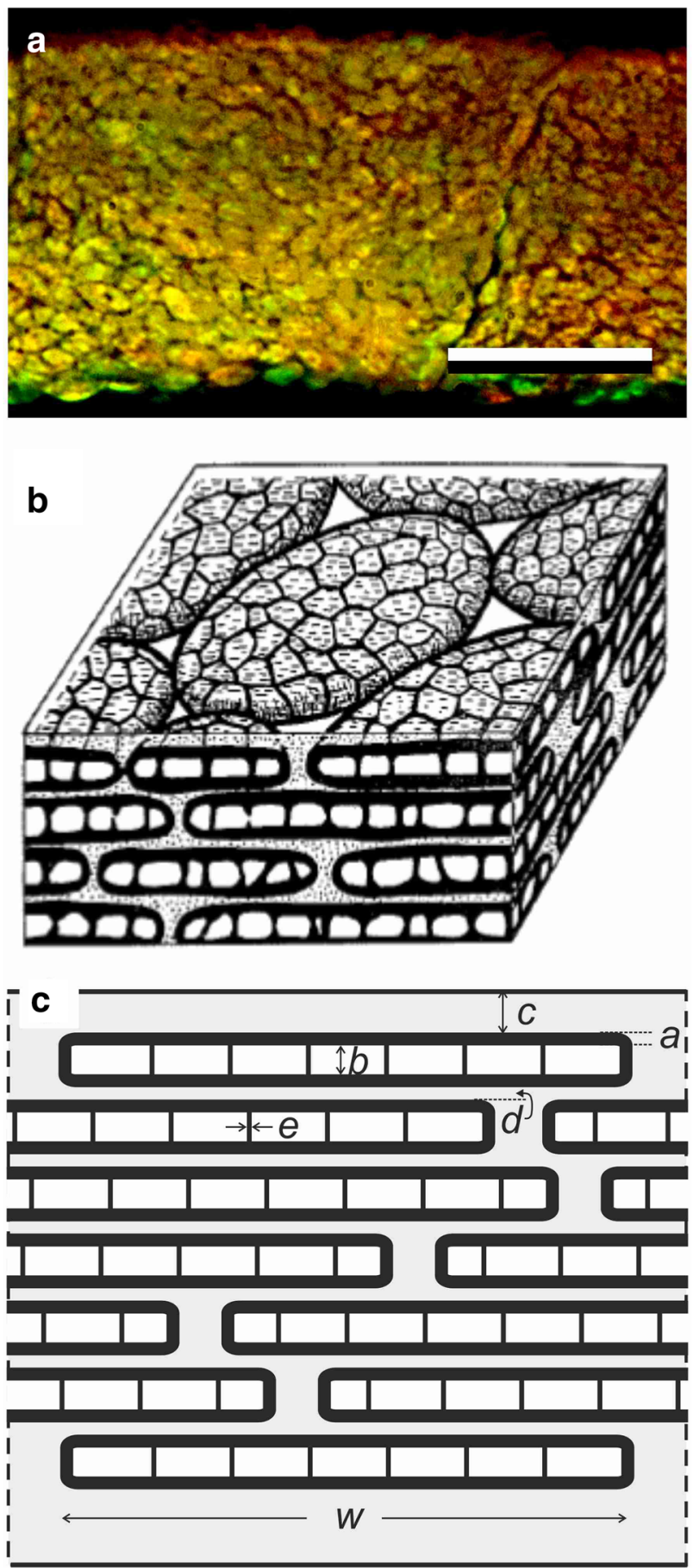

Fig. 6 Melanosome stacking in hummingbird barbules. a Epi-illumination micrograph of a barbule immersed in oil showing 1.5 cells; scale bar: $10 \mu \mathrm{m}$. b Drawing based on TEM anatomy (Fig. 42 from Durrer 1977). c Diagram showing the parameters used in the modeling (see "Materials and methods")

therefore, we considered case 2, where first the refractive index values of the three top layers (the cortex, the upper membrane of the first melanosome layer and its thin air layer) were averaged and secondly the refractive index values of the two closely opposed membranes of adjacent melanosome layers together with the intermediate keratin layer were averaged (Fig. 7a). This resulted in reflectance and absorbance spectra with increased red peaks (Fig. 7c, e). Apparently the refractive index jump at the barbule surface plays a crucial role, because when we neglected the top layers (case 3, Fig. 7a), the red peaks of both the reflectance and absorbance increased substantially (Fig. 7c, e).

The refractive index profile of case 3 resembles a square wave, except for the last bit of membrane (Fig. 7a). We, therefore, considered case 4 , which has 12 square waves with extreme values identical to those of the square wave of case 3. Image analysis of the electron micrographs indicated that a sinusoidally varying refractive index may be a more realistic representation of the barbule's refractive index profile. For instance, the barbule surface is slightly corrugated and the upper melanosome layer is not homogeneously filled with melanosomes (Fig. 5d-f), and hence the effective refractive index will not change abruptly in the barbule surface layer. The melanosomes are also not arranged in perfectly flat layers, which will cause smoothing of the refractive index profile. We, therefore, compared case 4 with case 5, a sine wave (Fig. 7b). The reflectance and absorbance spectra calculated for both cases appeared to be rather similar, except for a slight difference in magnitude (Fig. 7d, f).

The prominent reflectance band in the modeled spectra in the red wavelength range resembles the main band in the measured reflectance spectra (Fig. 1e), but the sideband in the blue wavelength range of the latter spectra was not reproduced. As the top melanosome layer strongly differed from the other layers, we investigated the possibility that the shape of the reflectance spectra is subtly sensitive to the properties of the first melanosome layer. We varied the thickness of the first air layer $b_{1}$ from the initially chosen value of $50 \mathrm{~nm}$. A distinct reflectance valley emerged at decreasing $b_{1}$ values (Fig. 8a). Assuming thus $b_{1}=25 \mathrm{~nm}$, this valley appeared to be insensitive to the thickness of the air layer in the inner melanosomes, $b_{2}$, when varied from 110 to $125 \mathrm{~nm}$ (Fig. 8b).

We furthermore investigated the dependence of the reflectance on the number of periods (i.e. layers), using the values $b_{1}=25 \mathrm{~nm}$ and $b_{2}=110 \mathrm{~nm}$. The reflectance bandwidth is large with only a few melanosome layers but rapidly narrows with an increase in layer number (Fig. 8c). With more than 10 layers the peak reflectance stabilizes and the bandwidth of $\sim 100 \mathrm{~nm}$ corresponds well with the experimental data. This is classical behaviour for biological multilayer reflectors (Land 1972; Wilts et al. 2009; Stavenga et al. 2017). We finally calculated the dependence of the reflectance spectra on the angle of light incidence for the case with 12 periods. Averaging the spectra for TE- and TM-polarized light yielded the reflectance spectra for unpolarized light. With an increasing angle of light incidence, the red peak strongly shifted towards shorter wavelengths (Fig. 8d). 
Fig. 7 Modeling the optical properties of a barbule. $\mathbf{a}, \mathbf{b}$ Real and imaginary parts of the effective refractive index (RI) profile at $500 \mathrm{~nm}, \operatorname{Re}\left(n_{\text {eff }}\right)$ and $\operatorname{Im}\left(n_{\text {eff }}\right)$. c, $\mathbf{d}$ Reflectance spectra resulting for the cases $1-5$ for normally incident light. $\mathbf{e}, \mathbf{f}$ Absorbance spectra resulting for cases 1-5. Case 1: A stack of 12 melanosomes. The RI is high at the melanosome membrane level, lower at the level of the keratin intermediate of the melanosomes, and much lower at the level of the air compartments of the melanosomes. Case 2: The RIs of two adjacent membranes and the intermediate keratin are averaged, and also the average is taken of the RIs in the frontal part, existing of the cortex layer, the distal membrane as well as the layer of the inner part of the upper melanosome. Case 3: The frontal part is neglected, resulting in 11 periods of a square wave plus a final RI pulse of a membrane layer. Case 4: Similar to case 3, except for the replacement of the last membrane layer by another square wave period. Case 5: A sinusoidally oscillating RI with extreme values identical to those of case 4
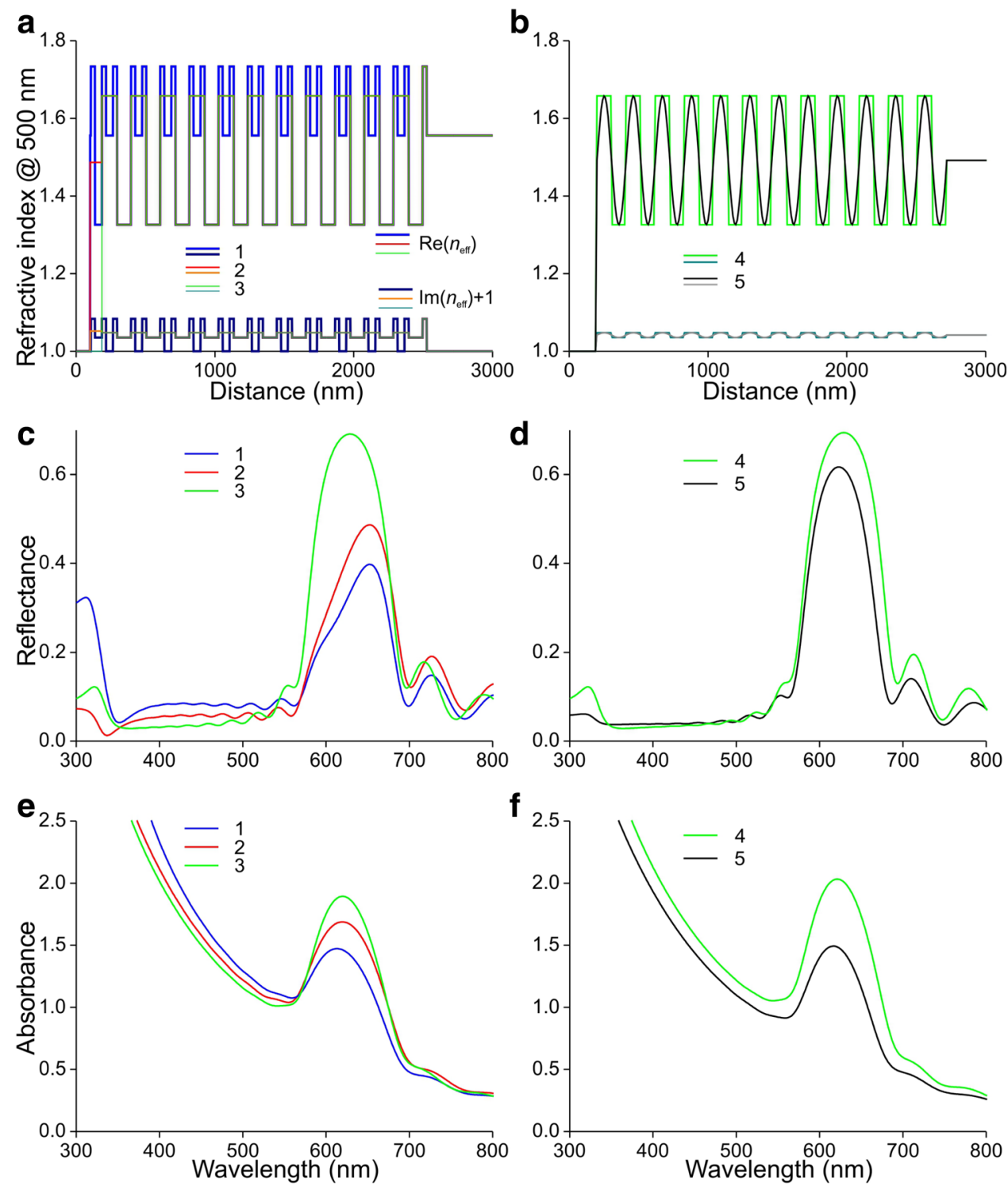

\section{Discussion}

We have modeled the reflectance of the feather barbules of the male Anna's hummingbird's gorget using a transfer matrix method for optical multilayers. We assumed dimensions suggested by anatomy, and we estimated the effective refractive indices of the layers via a simple, weighted average of the refractive indices of the material components. The applied approach allows considerable insight into the essential optical properties of the barbules of hummingbird feathers. For instance, the strong hypsochromic shift occurring upon an increase of the angle of light incidence following from the model calculations (Fig. 8d) is fully in agreement with measured spectra (Meadows et al. 2011) as well as with the colour change from red via yellow to greenish seen in the scatterograms when changing the angle of illumination from normal to $50^{\circ}$ (Fig. 2). Furthermore, the calculations show that a stack of more than 10 melanosome layers of the hummingbird barbules creates a high peak reflectance, close to saturation (Fig. 8c). Adding more layers only very slightly increases the peak reflectance and narrows the bandwidth. This will only make sense when extreme brilliance and spectral contrast is very important for signaling, which is apparently the case, given the $12-15$ layers seen in the electron micrographs (Fig. 5d-f).

The effective refractive indices used in the modelling were estimated via a simple volume average of the components. The large differences in the refractive index values between melanin, keratin and air may yield slightly deviant effective refractive indices, but the modeled reflectance spectra will be appropriate approximations, certainly compared to the modeled spectra of Greenewalt et al. (1960a, b), who in their pioneering studies on the structural colouration of hummingbird feathers assumed a wavelength independent, 
Fig. 8 Reflectance spectra of a barbule with various parameters. a Dependence of the reflectance on the thickness of the top melanosome layer; normally incident light, 12 layers $\left(b_{1}\right.$ : thickness of air layer of first melanosome layer; $b_{2}$ : thickness of air layer of the second to last melanosome layer). b Dependence of the reflectance on the thickness of the melanosome layers below the top layer. c Dependence of the reflectance on the number of periods (layers). d Dependence of the reflectance on the angle of light incidence of unpolarized light; 12 layers (c, d: $b_{1}=25 \mathrm{~nm}$, $b_{2}=110 \mathrm{~nm}$ )
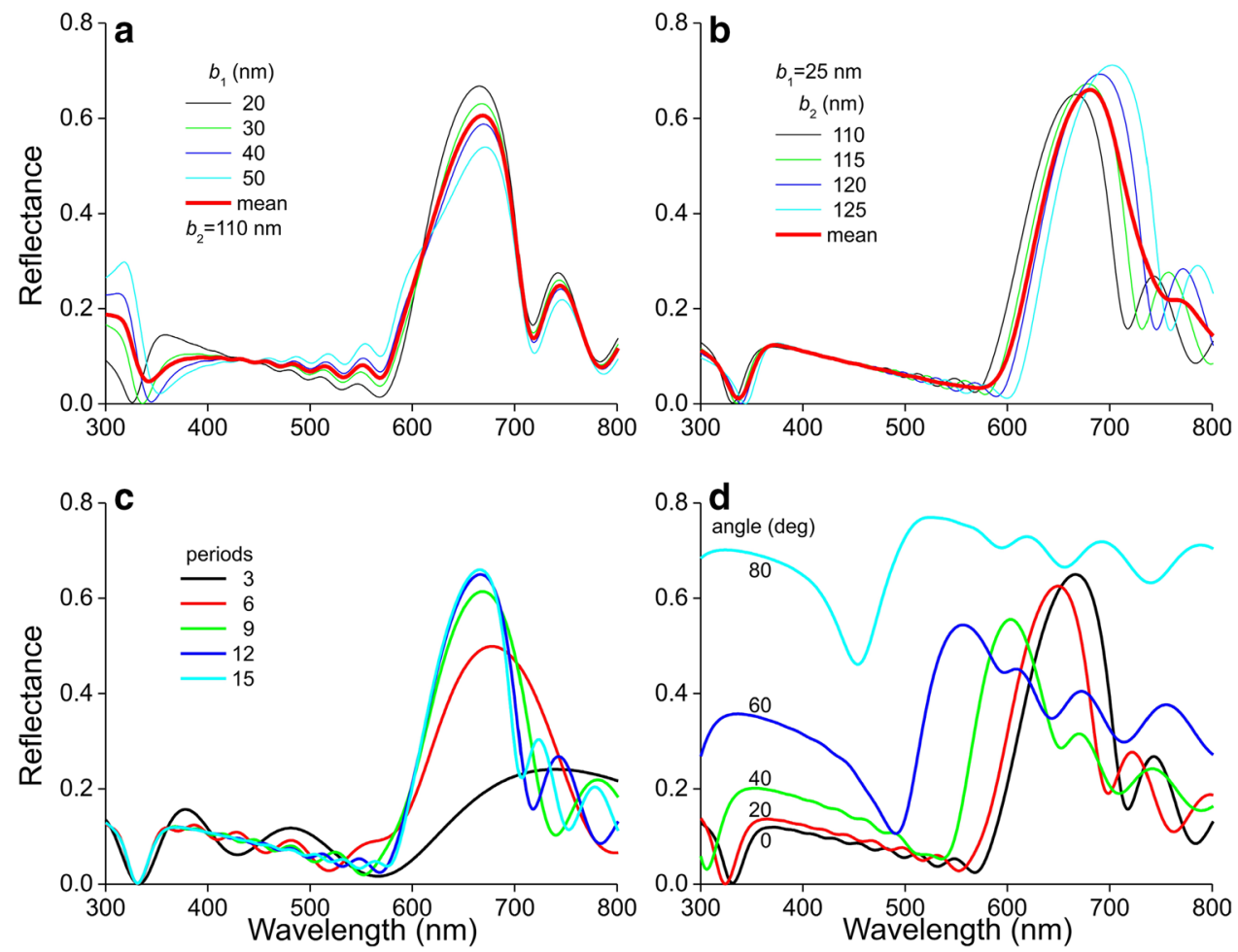

real refractive index of melanin $\left(n_{\mathrm{m}}=2.0\right)$, which we now know is not close to realistic values (Stavenga et al. 2015).

Quite critical are the dimensional values used in the calculations. For instance, a sinusoidal refractive index profile with period 210,220 or $230 \mathrm{~nm}$ produces reflectance spectra with peak wavelengths 623, 652, and $680 \mathrm{~nm}$, respectively. The experimentally obtained reflectance peak wavelength of the gorget feathers was $\sim 670 \mathrm{~nm}$, which indicates that the period $210 \mathrm{~nm}$ chosen on the basis of anatomical melanosome values, represents a slight underestimate. Generally, hummingbirds display structural colours varying throughout the whole visible wavelength range, from violet-blue to scarlet-red. This can be straightforwardly achieved by adjusting the periodicity of the barbules' melanosome stacks (Giraldo et al. in prep.).

In our optical modelling, we have not taken into account the space below the melanosome stack. Part of the incident light that has passed the melanosomes will reach the lower surface of the barbule speculum, where it may be partly reflected. In principle that reflected light could contribute to the interference process, but anatomy shows that the lower surface of the barbule is far from flat, so that the reflected light will not substantially contribute to interference, as well as will be spread into a large spatial angle. The reflected light flux can, therefore, be neglected, especially in the shorter wavelength range where melanin absorption is high. In the very long-wavelength range, where melanin absorption is minor, scattering by the randomly arranged melanosomes may contribute a slight background reflectance.
Similar as Greenewalt et al. (1960a), we found that barbules in immersion oil had reflectance spectra with only the main red peak, but no blue secondary band. The modeling of Fig. 8 shows that the top layer with very thin melanosomes plays a crucial role in creating the sideband. A valley in the reflectance spectra emerges when the air layer is assumed to be very minor. However, the calculated blue sideband extends well into the ultraviolet, not completely corresponding with the measured spectra. We hence have not yet reached a fully satisfactory modeled reflectance in the blue wavelength range. We nevertheless believe that the main features of the $C$. anna hummingbird feathers can now be understood in considerable detail.

The structural coloured feathers of hummingbirds are highly specialized. As noted by Durrer (1977), the barbules of bird feathers are usually oriented perpendicular to the feather plane, probably to optimise hydrophobicity (Eliason and Shawkey 2011). To display a structural-coloured barbule, it must be rotated so that it becomes more or less coplanar with the feather surface. Durrer (1977) provides many examples of structural-coloured bird barbules that are curved, but the male Anna's hummingbird's barbules appear to be hybrid. They are folded planes, of which the speculum part is about parallel to the feather plane and the other, the velum part, has maintained the about normal, perpendicular orientation (Greenewalt et al. 1960a; Durrer 1977). This allows the barbules of adjacent barbs to preserve their interconnections via the hooks (Durrer 1977). Whereas the velum part is hidden, only the 
speculum part of the barbule is exposed and thus can have a display function.

The highly specialized features of structurally coloured feathers in birds are assumed to be a product of sexual and natural selection (Darwin 1871; Parra 2010; Prum 2012). One of the most famous examples of the use of structural colouration signals in courtship comes from birds of paradise (family: Paradisaeidae). The barbules of the breast feathers of the male bird of paradise Parotia lawesii are boomerang-shaped, and the cortex is a blue-reflecting thin film enveloping a golden-yellow reflecting multilayer of rod-shaped melanosomes (Stavenga et al. 2010; Wilts et al. 2014). During courtship, the male flashes the highly reflecting breast feathers as well as nape feathers towards on-looking females that are seated on an overhanging branch (Laman and Scholes 2012).

Male Anna's hummingbirds and other close relatives do it quite differently. The male uses two flight displays: dives and shuttles (Stiles 1982). While the former is the most widely known (Clark 2009, 2012), the second seems to be the most critical during courtship (Stiles 1982). During the shuttle display the male often hovers close $(<30 \mathrm{~cm})$ and obliquely above a female with his head pointed towards her, so that the angled barbules maximally reflect incident sky and/or sunlight, spectrally reduced into a narrow wavelength band, toward the female (Stiles 1982, but see also; Simpson and McGraw 2018; https://www.youtube.com/watch?v=scCZ8 aoq_sA). It is important to notice then that the reflectance band displayed toward the female is shifted to shorter wavelengths, depending on the angle of light incidence (and reflection); the spectral shift becomes quite noticeable at angles of incidence $>20^{\circ}$ (Fig. 8d). Presumably, the reason why the barbules' speculae are not fully parallel to the feather plane, but rather organized like a closely fitting Venetian blind, is because this way the sky and sun light's angles of incidence and reflection are reduced, and thus the peak wavelength of the reflected light will remain above $600 \mathrm{~nm}$.

The reflected light spectrum thus about coincides with the spectral sensitivity of the visual system's red receptor, because similar to other birds, hummingbird vision is most likely tetrachromatic, based on a set of UVS/VS, SWS, MWS and LWS receptors. The peak wavelength of the LWS receptors of all 21 species studied by Hart and Vorobyev (2005) was $>600 \mathrm{~nm}$. Herrera et al. (2008) derived from ERG measurements a peak wavelength $560 \mathrm{~nm}$ for the LWS receptor of the green-backed firecrown hummingbird, Sephanoides sephaniodes, but that value is presumably an underestimate. A potential advantage of a structure that generates a reflectance spectrum that peaks at a very long wavelength and has a narrow bandwidth is the high chromatic contrast with the background, which has been shown to be used by birds when searching for food (Schaefer et al. 2006) and also by red flowers against their background (Herrera et al. 2008).
Thus, this might be another example of sensory exploitation in which the male captures the female's attention.

Acknowledgements This study was financially supported by the Colombian Administrative Department for Science and Technology Colciencias (Grant code 111571250482-contract number 248-2016 to J.L.P.) and the Air Force Office of Scientific Research/European Office of Aerospace Research and Development AFOSR/EOARD (Grant FA9550-15-1-0068, to D.G.S.). We thank the Museum of Vertebrate Zoology (UC Berkeley) for collaboration and three anonymous reviewers for critical comments and suggestions for improvements.

Open Access This article is distributed under the terms of the Creative Commons Attribution 4.0 International License (http://creativeco mmons.org/licenses/by/4.0/), which permits unrestricted use, distribution, and reproduction in any medium, provided you give appropriate credit to the original author(s) and the source, provide a link to the Creative Commons license, and indicate if changes were made.

\section{References}

Clark CJ (2009) Courtship dives of Anna's hummingbird offer insights into flight performance limits. Proc R Soc B 276:3047-3052

Clark CJ (2012) The role of power versus energy in courtship: what is the 'energetic cost' of a courtship display? Anim Behav 84:269-277

Cuthill IC, Allen WL, Arbuckle K, Caspers B, Chaplin G, Hauber ME, Hill GE, Jablonski NG, Jiggins CD, Kelber A, Mappes J, Marshall J, Merrill R, Osorio D, Prum R, Roberts NW, Roulin A, Rowland HM, Sherratt TN, Skelhorn J, Speed MP, Stevens M, Stoddard MC, Stuart-Fox D, Talas L, Tibbetts E, Mappes J (2017) The biology of color. Science 357:eaan0221

Darwin C (1871) The descent of man, and selection in relation to sex. John Murray, London

Doucet SM, Meadows MG (2009) Iridescence: a functional perspective. J R Soc Interface 6:S115-S132

Durrer H (1977) Schillerfarben der Vogelfeder als Evolutionsproblem. Denkschr Schweiz Naturforsch Ges 91:1-126

Eliason CM, Shawkey MD (2011) Decreased hydrophobicity of iridescent feathers: a potential cost of shiny plumage. J Exp Biol 214:2157-2163

Greenewalt CH (1960) Hummingbirds. Dover, New York

Greenewalt CH, Brandt W, Friel DD (1960a) Iridescent colors of hummingbird feathers. J Opt Soc Am 50:1005-1013

Greenewalt CH, Brandt W, Friel DD (1960b) The iridescent colors of hummingbird feathers. Proc Am Philos Soc 104:249-253

Hart NS, Vorobyev M (2005) Modelling oil droplet absorption spectra and spectral sensitivities of bird cone photoreceptors. J Comp Physiol A 191:381-392

Herrera G, Zagal JC, Diaz M, Fernández MJ, Vielma A, Cure M, Martinez J, Bozinovic F, Palacios AG (2008) Spectral sensitivities of photoreceptors and their role in colour discrimination in the green-backed firecrown hummingbird (Sephanoides sephaniodes). J Comp Physiol A 194:785-794

Laman T, Scholes E (2012) Birds of paradise: revealing the world's most extraordinary birds. National Geographic Society, Washington D.C.

Land MF (1972) The physics and biology of animal reflectors. Prog Biophys Mol Biol 24:77-105

Leertouwer HL, Wilts BD, Stavenga DG (2011) Refractive index and dispersion of butterfly scale chitin and bird feather keratin measured by interference microscopy. Opt Express 19:24061-24066 
Meadows MG, Morehouse NI, Rutowski RL, Douglas JM, McGraw KJ (2011) Quantifying iridescent coloration in animals: a method for improving repeatability. Behav Ecol Sociobiol 65:1317-1327

Meadows MG, Roudybush TE, McGraw KJ (2012) Dietary protein level affects iridescent coloration in Anna's hummingbirds, Calypte anna. J Exp Biol 215:2742-2750

Parra JL (2010) Color evolution in the hummingbird genus Coeligena. Evolution 64:324-335

Prum RO (2012) Aesthetic evolution by mate choice: Darwin's really dangerous idea. Philos Trans R Soc Lond B 367:2253-2265

Schaefer HM, Levey DJ, Schaefer V, Avery ML (2006) The role of chromatic and achromatic signals for fruit detection by birds. Behav Ecol 17:784-789

Simpson RK, McGraw KJ (2018) Two ways to display: male hummingbirds show different color-display tactics based on sun orientation. Behav Ecol 29:637-648

Stavenga DG (2014) Thin film and multilayer optics cause structural colors of many insects and birds. Mater Today Proc 1S:109-121

Stavenga DG, Leertouwer HL, Pirih P, Wehling MF (2009) Imaging scatterometry of butterfly wing scales. Opt Express 17:193-202

Stavenga DG, Leertouwer HL, Marshall NJ, Osorio D (2010) Dramatic colour changes in a bird of paradise caused by uniquely structured breast feather barbules. Proc R Soc B 278:2098-2104
Stavenga DG, Leertouwer HL, Hariyama T, De Raedt HA, Wilts BD (2012) Sexual dichromatism of the damselfly Calopteryx japonica caused by a melanin-chitin multilayer in the male wing veins. PLoS One 7:e49743

Stavenga DG, Leertouwer HL, Osorio DC, Wilts BD (2015) High refractive index of melanin in shiny occipital feathers of a bird of paradise. Light Sci Appl 4:e243

Stavenga DG, Meglič A, Pirih P, Koshitaka H, Arikawa K, Wehling MF, Belušič G (2017) Photoreceptor spectral tuning by colorful, multilayered facet lenses in long-legged fly eyes (Dolichopodidae). J Comp Physiol A 203:23-33

Stiles FG (1982) Aggressive and courtship displays of the male Anna's hummingbird. Condor 84:208-225

Stoddard MC, Prum RO (2011) How colorful are birds? Evolution of the avian plumage color gamut. Behav Ecol 22:1042-1052

Wilts BD, Leertouwer HL, Stavenga DG (2009) Imaging scatterometry and microspectrophotometry of lycaenid butterfly wing scales with perforated multilayers. J R Soc Interface 6:S185-S192

Wilts BD, Michielsen K, De Raedt H, Stavenga DG (2014) Sparkling feather reflections of a bird-of-paradise explained by finitedifference time-domain modeling. Proc Natl Acad Sci USA 111:4363-4368 